\title{
Amnestic Mild Cognitive Impairment Is Associated With Frequency-Specific Brain Network Alterations in Temporal Poles
}

\author{
Francesca Jacini 1,2*†, Pierpaolo Sorrentino ${ }^{3,4 \dagger}$, Anna Lardone 1,2, Rosaria Rucco ${ }^{1,2}$, \\ Fabio Baselice ${ }^{3}$, Carlo Cavaliere ${ }^{5}$, Marco Aiello ${ }^{5}$, Mario Orsini ${ }^{5}$, Alessandro lavarone ${ }^{6}$, \\ Valentino Manzo ${ }^{7}$, Anna Carotenuto ${ }^{7}$, Carmine Granata $^{8}$, Arjan Hillebrand ${ }^{4}$ \\ and Giuseppe Sorrentino ${ }^{1,2}$
}

\begin{abstract}
'Department of Motor Sciences and Wellness, Parthenope University of Naples, Naples, Italy, ${ }^{2}$ Institute for Diagnosis and Cure Hermitage Capodimonte, Naples, Italy, ' Department of Engineering, Parthenope University of Naples, Naples, Italy, ${ }^{4}$ Department of Clinical Neurophysiology and MEG Center, VU University Medical Center Amsterdam, Amsterdam, Netherlands, ${ }^{5}$ Diagnostic and Nuclear Research Institute, IRCCS SDN, Naples, Italy, ${ }^{6}$ Neurological and Stroke Unit, CTO Hospital-AORN Ospedale dei Colli, Naples, Italy, ${ }^{7}$ Department of Neurology, AORN Cardarelli, Naples, Italy, ${ }^{8}$ Institute of Applied Sciences and Intelligent Systems, CNR, Pozzuoli, Italy
\end{abstract}

OPEN ACCESS

Edited by:

Guido Gainotti,

Università Cattolica del Sacro Cuore,

Italy

Reviewed by:

Ricardo Bruña,

Complutense University of Madrid,

Spain

Marie-Constance Corsi, Institut National de Recherche en Informatique et en Automatique (INRIA), France

${ }^{*}$ Correspondence: Francesca Jacini francesca.jacini@uniparthenope.it

${ }^{\dagger}$ These authors have contributed equally to this work

Received: 05 July 2018 Accepted: 20 November 2018 Published: 06 December 2018

Citation: Jacini F, Sorrentino P, Lardone A, Rucco R, Baselice F, Cavaliere C, Aiello $M$, Orsini $M$, lavarone $A$, Manzo V, Carotenuto A, Granata C, Hillebrand $A$ and Sorrentino $G$ (2018) Amnestic Mild Cognitive Impairment Is Associated With

Frequency-Specific Brain Network Alterations in Temporal Poles. Front. Aging Neurosci. 10:400. doi: 10.3389/fnagi.2018.00400
There is general agreement that the neuropathological processes leading to Alzheimer's disease (AD) begin decades before the clinical onset. In order to detect early topological changes, we applied functional connectivity and network analysis to magnetoencephalographic (MEG) data obtained from 16 patients with amnestic Mild Cognitive Impairment (aMCI), a prodromal stage of $\mathrm{AD}$, and 16 matched healthy control (HCs). Significant differences between the two groups were found in the theta band, which is associated with memory processes, in both temporal poles (TPs). In aMCl, the degree and betweenness centrality (BC) were lower in the left superior TP, whereas in the right middle TP the $\mathrm{BC}$ was higher. A statistically significant negative linear correlation was found between the BC of the left superior TP and a delayed recall score, a sensitive marker of the "hippocampal memory" deficit in early AD. Our results suggest that the TPs, which are involved early in AD pathology and belong to the memory circuitry, have an altered role in the functional network in aMCl.

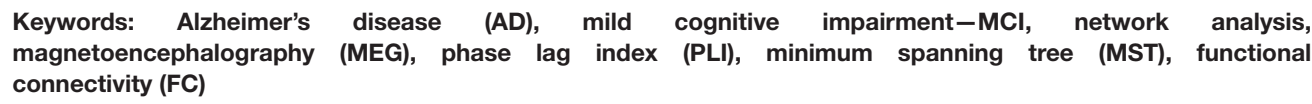
magnetoencephalography (MEG), phase lag index (PLI), minimum spanning tree (MST), functional connectivity (FC)

\section{INTRODUCTION}

A large number of studies have investigated the causes of Alzheimer's disease (AD); nevertheless, many uncertainties remain about its pathophysiology (Kanfer et al., 1999; Sorrentino et al., 2014). It is likely that multiple mechanisms contribute to the deposits of senile plaques and neurofibrillary tangles, the hallmarks of $\mathrm{AD}$ pathology (Sorrentino et al., 2008). It is well established that the spatiotemporal pattern of progression of neurofibrillary degeneration starts in the allocortex of the medial temporal lobe (entorhinal cortex and hippocampus) and spreads

Abbreviations: BC, betweenness centrality; MEG, magnetoencephalography; MST, minimum spanning tree; PLI, phase lag index; TP, temporal pole. 
to the associative neocortex (Braak and Braak, 1991; Braak et al., 2006). Furthermore, it is now a general understanding that the neuropathological processes leading to $\mathrm{AD}$ begin decades before the onset of clinical symptoms (Sorrentino and Bonavita, 2007; Jack et al., 2010).

Mild Cognitive Impairment (MCI) has been characterized by an objective cognitive impairment in a given domain, mainly memory, not yet implying the definition of dementia (Petersen et al., 1999). Yet, MCI is associated with a higher risk of developing dementia, including AD (Shah et al., 2000; Petersen et al., 2009). In recent years, this definition underwent significant evolution. The original notion of a predominant amnestic problem was substituted by a new perspective where different clinical subtypes were defined. Nowadays, MCI patients are categorized according to type and number of affected cognitive domains. This clinical classification is particularly relevant because each subtype is linked to a presumed etiology, with the amnestic subtypes (aMCI) considered as a prodromal form of AD (Petersen et al., 2009, 2014).

The human brain can be seen as a complex system characterized by a balancing between integration and segregation mechanisms through spatial and temporal interaction of distinct neuronal populations (Sporns et al., 2005; Lopes da Silva, 2013; Sporns, 2013). One of the strategies to estimate the interactions between brain areas, referred to as "functional connectivity" (Friston, 2011), is to exploit statistical dependencies that might be present between the time series of neuronal activation in these areas. Brain networks can be characterized using a combination of graph theory and modern network science, applied to neuroimaging data obtained from techniques such as functional magnetic resonance imaging (fMRI; Stam, 2010; Wang, 2010). To enable characterization of the macroscopic brain network topology using graph analysis, brain areas are typically used as nodes and the interactions between the brain areas as links.

It has been proposed that in neurodegenerative diseases, the spreading of the pathological process reflects itself in the reduction of structural and functional connectivity (Trojsi et al., 2017), and it has been suggested that $A D$ may be regarded as a disconnection syndrome (Pievani et al., 2011; Minati et al., 2013; Stam, 2014). Previous studies showed that the network architecture of AD brains loses the typical "small world" organization (Watts and Strogatz, 1998), disrupting the balance between functionally highly specialized areas and long-range interactions between distant regions (Stam et al., 2009; SanzArigita et al., 2010; Stam, 2010). There is also some evidence that the most connected nodes are preferentially affected by pathological processes (Buckner et al., 2009; Stam, 2014) perhaps due to their high metabolic activity (de Haan et al., 2012). Starting from there, the functional disconnection might spread to other brain regions (Brier et al., 2014). Possibly, damage to a node could lead to overload, and subsequent failure, of the hierarchically upstream nodes. This could be the mechanism underlying the spreading of neurodegeneration (Stam, 2014). Hence, in order to capture the subtle changes underlying cognitive impairment, we need to evaluate the brain as a complex system (Sporns, 2011).
There is ample evidence that a temporally and spatially balanced pattern of synchronized and desynchronized oscillations in distinct frequency bands underlies specific brain functions, including cognitive functions such as language, memory, thought or awareness (Gross et al., 2004). Beside fMRI, relevant information on the functioning of brain networks can be retrieved using neurophysiological techniques, such as electroencephalography (EEG) and magnetoencephalography (MEG), since they directly capture the oscillatory activity of the neuronal ensembles, thereby providing clinical and pathophysiological information about brain functioning in health and disease (Lopes da Silva, 2013). While retaining the high temporal resolution of EEG, MEG signals are not distorted by the layers surrounding the brain, allowing for a temporally and spatially precise reconstruction of the neural activity within the brain (Baillet, 2017). Furthermore, the high temporal resolution of MEG signals allows for a more sophisticated estimation of synchronization between brain areas. For example, the phase lag index (PLI; Stam et al., 2007) is a phase-based metric (insensitive to volume conduction/field spread) that quantifies phase-synchronization (Rosenblum et al., 1996) between areas, rather than the simultaneous amplitude fluctuations typically estimated with fMRI.

Several MEG studies have addressed network changes in $\mathrm{AD}$, but the results have not always been consistent (for an extensive review see Engels et al., 2017). The reason for the non-homogeneity of findings may have its origin in both the clinical features of the examined populations and the analysis methods. $\mathrm{AD}$ has a long clinical course linked to a well-defined neuropathological evolution (Braak and Braak, 1991, 1995, 1996). As a consequence, the disease stage at which the recording takes place may be crucial. Another possible source of inconsistency of the experimental observations may lie in the fact that network reconstructions may be biased (van Wijk et al., 2010). In particular, one main problem is the comparison of networks with different edge densities, average degrees, or edge weights. Normalization of the data or selection of arbitrary cut-offs does not solve this issue (van Wijk et al., 2010; Otte et al., 2015). A possible approach to overcome these limitations is the use of the minimum spanning tree (MST; Stam et al., 2014). Starting from the full network, the MST allows for the construction of a unique sub-graph that connects all the nodes without forming cycles. This way, it is possible to identify the backbone of the original network. If the link weights are unique, this procedure provides a unique reconstruction of networks with the same number of nodes and links. The metrics calculated on the MST capture information of the original network (Tewarie et al., 2015) while allowing the unbiased statistical comparisons of those metrics across groups, in the sense that observed topological differences are not trivially due to differences in functional connectivity (van Wijk et al., 2010).

The aim of our study is to identify subtle changes in network topology in aMCI. Given that aMCI is considered a prodromal stage of $\mathrm{AD}$, our hypothesis is that in aMCI the topological alterations of the network involve brain areas 
that are known to degenerate early in AD. Furthermore, we hypothesized that such alterations might be specific to brain rhythms related to memory processes. Detecting subtle functional alterations might enable the identification of early disease markers and to shed light on the pathophysiological mechanisms of neurodegeneration. To test our hypothesis, we applied the PLI, followed by the MST, to MEG data obtained from a cohort of aMCI patients and healthy controls (HCs), and compared the reconstructed brain networks between these groups.

\section{MATERIALS AND METHODS}

\section{Participants}

Fifty-two participants, aged $65-80$ years, were screened. All subjects were right handed and native Italian speakers. Exclusion criteria were the presence of neurological or systemic illness that could affect the cognitive status, and contraindications to MRI or MEG recording. Based on neurological examination and extensive neuropsychological assessment (see Table 1), subjects were divided into two groups: $21 \mathrm{MCI}$ and 31 controls. MCI diagnosis was formulated according to the National Institute on Aging-Alzheimer Association (NIA-AA) criteria (Albert et al., 2011), which include: (i) cognitive concern reported by patient or informant or clinician; (ii) objective evidence of impairment in one or more cognitive domains, typically including memory; (iii) preservation of independence in functional abilities; and (iv) not demented. Three of the MCI patients were non-amnestic MCI so they were excluded from the study. Reduced hippocampal volume detected by structural MRI (see Table 2), a neuronal injury marker, gives our MCI cohort an intermediate likelihood of

TABLE 1 | Neuropsychological evaluation.

\begin{tabular}{ll}
\hline Test & Explored function \\
\hline MMSE & Global cognitive status \\
FAB & Frontal efficiency \\
FCSRT & \\
FCSRT immediate free recall & \\
FCSRT immediate total recall & "Hippocampal" episodic memory \\
FCSRT delayed free recall & \\
FCSRT delayed total recall & \\
FCSRT index of sensitivity of cueing & \\
MDB & \\
Rey's 15 word immediate recall & Short and long-term verbal \\
Rey's 15 word delayed recall & episodic memory \\
Word fluency & Ability to access lexical-semantic \\
Phrase construction & memory store \\
Raven's 47 progressive matrices & Language \\
Immediate visual memory & Conceptual reasoning \\
& Short-term \\
Freehand copying of drawings & visuoperceptual recognition \\
Copying drawings with landmarks & memory \\
BDI & Constructive praxia \\
\hline
\end{tabular}

MMSE, Mini Mental State Examination (Measso et al., 1993); FAB, Frontal Assessment Battery (lavarone et al., 2004); FCSRT, Free and Cued Selective Reminding Test (Frasson et al., 2011); MDB, Mental Deterioration Battery (Carlesimo et al., 1996); BDI, Beck Depression Inventory (Sica and Ghisi, 2007). being due to $\mathrm{AD}$ (Albert et al., 2011). Two patients and 15 controls did not complete the MRI because of difficulty in lying down or refusal to perform it, so they were excluded.

The subjects included in the study were 16 patients affected by aMCI (mean age 71, 7 years; standard deviation (SD). 6, 6; eight men and eight women) compared to 16 age, educational level and gender matched HC subjects (mean age 70, 3 years; SD 4, 2; nine man and seven women). The study was approved by the Local Ethics Committee "Comitato Etico Campania Centro" (Prot.n.93C.E./Reg. n.14-17OSS), and all subjects had given written informed consent. All methods included in the protocol were carried out in accordance with the Declaration of Helsinki.

\section{Magnetic Resonance Imaging}

MR images were acquired using a 3T Biograph mMR tomograph (Siemens Healthcare, Erlangen, Germany) equipped with a 12 channels head coil. The scan performed either after the MEG recording or a minimum of 21 days earlier (within 1 month). The following protocol was applied: (i) three-dimensional T1-weighted Magnetization-Prepared Rapid Acquisition Gradient-Echo sequence (MPRAGE, 240 sagittal planes, $214 \times 21 \mathrm{~mm}^{2}$ Field of View, voxel size $1 \times 1 \times 1 \mathrm{~mm}^{3}$, TR/TE/TI 2,400/2.5/1,000 ms, flip angle $8^{\circ}$ ); (ii) Three-dimensional T2-weighted Sampling Perfection with Application optimized Contrasts using different flip angle Evolution sequence (SPACE, 240 sagittal planes, $214 \times 214 \mathrm{~mm}^{2}$ Field of View, voxel size $1 \times 1 \times 1 \mathrm{~mm}^{3}$, TR/TE 3,370/563); (iii) Two-dimensional T2weighted turbo spin echo Fluid Attenuated Inversion Recovery sequence (FLAIR, 44 axial planes, $230 \times 230 \mathrm{~mm}^{2}$ Field of View, voxel size $0.9 \times 0.9 \times 0.9 \mathrm{~mm}^{3}$, TR/TE/TI 9,000/95/25,00, flip angle $150^{\circ}$ ). Volumetric analysis was performed with the Freesurfer software (version 6.0; Fischl et al., 2002). Volumes were normalized for the estimated total intracranial volume (eTIV). Vascular burden was assessed using Fazekas scale (Fazekas et al., 1987).

\section{MEG Acquisition}

The MEG system, equipped with 163 magnetometers, was developed by the National Research Council, Pozzuoli, Naples, at the Institute of Applied Sciences and Intelligent Systems "E. Caianiello" (Rombetto et al., 2014). Using Polhemus (Polhemus FASTRAK ${ }^{\circledR}$ ) we determined the location of four coils, placed on the forehead and behind the ears of the participants, and of four reference points on the head (nasion, right and left preauricular points, vertex). The head movement were evaluated by visual inspection through a camera placed inside the cabin. The coils were activated, and localized, at the beginning of each segment of the registration. Participants were seated inside a magnetically shielded room to reduce background noise (Advanced Technologies Biomagnetics, Ulm, Germany). Electrocardiographic (ECG) and Electrooculographic (EOG) signals were co-recorded to aid artifact removal (Gross et al., 2013). Spontaneous brain activity was recorded for two sets of $2.5 \mathrm{~min}$, in resting-state, with eyes closed. Signals, after an anti-aliasing filter, were acquired at a sampling frequency of $1,024 \mathrm{~Hz}$. The signal was then filtered using a fourth order 
TABLE 2 | Subjects characteristics.

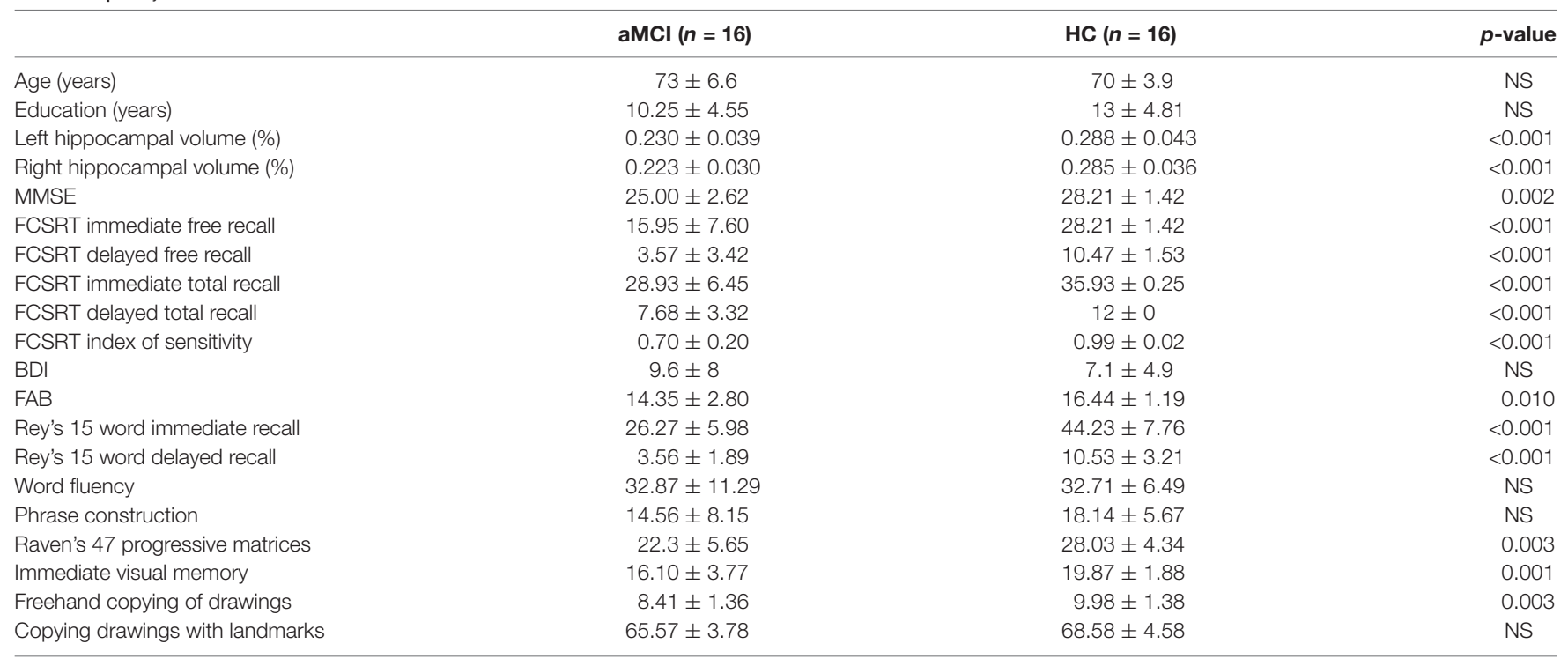

Demographic and clinical variables comparison in aMCl and control groups. Data are given as mean \pm standard deviation (SD). NS, not significant; aMCl, amnestic Mild Cognitive Impairment group; HC, healthy controls; MMSE, Mini Mental State Examination; FCSRT, Free and Cued Selective Reminding Test; BDI, Beck Depression Inventory; FAB, Frontal Assessment Battery.

Butterworth IIR band-pass filter in the $0.5-48 \mathrm{~Hz}$ band. In order to reduce the environmental noise, principal component analysis (PCA) was implemented (Sadasivan and Dutt, 1996). The methodology consists in computing the space base of the signals acquired by the reference sensors (i.e., environmental noise), and in projecting the signals from the brain sensors on such base in order to remove the noise parallel components from the brain signal (de Cheveigné and Simon, 2007). The PCA filtering implementation available within the Fieldtrip Toolbox (Oostenveld et al., 2011) was used. Subsequently, noisy channels (on average $14 \pm 5$ channels) were removed manually through visual inspection of the whole dataset by an experienced rater (Gross et al., 2013). Physiological artifacts, cardiac and blinking (if present), were removed from the signals through supervised independent component analysis (ICA). Typically, one component has been deleted for ECG and no component for EOG. The processing has been done using the Fieldtrip toolbox (version 2014.05.06; Oostenveld et al., 2011).

\section{Source Reconstruction}

The subject's fiducial points were visually identified on the native MRI of the subjects and used to coregister the MEG acquisition, whereupon the MRI was spatially normalized to an average T1-MRI template, part of the SPM toolbox ${ }^{1}$. Subsequently, we used the volume conduction model proposed by Nolte (2003) and we applied the Linearly Constrained Minimum Variance (LCMV) beamformer (Van Veen et al., 1997) to reconstruct the time series in the centroids of 116 regionsof-interest (ROIs). In order to compute the beamformer weights, we used the broad-band data covariance matrix and an identity noise covariance matrix (hence assuming

\footnotetext{
$\overline{{ }^{1} \text { http://imaging.mrc-cbu.cam.ac.uk/imaging/Templates }}$
}

uncorrelated noise). The optimal source orientation was found using a Singular Value Decomposition (SVD). The labeling of the regions was based upon the Automated Anatomical Labeling (AAL) atlas (Tzourio-Mazoyer et al., 2002; Gong et al., 2009). We considered only the first 90 ROIs, excluding those corresponding to the cerebellum given the low reliability of the reconstructed signal in those areas. For each region, we projected the time series along the dipole direction that explained most variance by mean of SVD using Fieldtrip.

The signals in the source space have been downsampled to $512 \mathrm{~Hz}$. By visual inspection, the first 10 epochs of $8 \mathrm{~s}$ for each subject that did not contain artifacts (either system related or physiological) or strong environmental noise were selected. All the source reconstruction processing has been performed in Matlab environment using the Fieldtrip toolbox.

\section{Functional Connectivity Analysis}

The functional connectivity analysis was performed using BrainWave software (version 0.9.152.4.1, available from https:/home.kpn.nl/stam7883/brainwave.html). The length of $8 \mathrm{~s}$ is a trade-off between the need to have enough cleaned epochs (Gross et al., 2013; Sorrentino et al., 2017) and to obtain a reliable estimate of the functional connectivity (Fraschini et al., 2014), while avoiding drowsiness. The epochs were band-pass filtered into five canonical frequency bands using Brainwave: delta $(0.5-4 \mathrm{~Hz})$, theta $(4-8 \mathrm{~Hz})$, alpha $(8-13 \mathrm{~Hz})$, beta $(13-30 \mathrm{~Hz})$ and gamma (30-48 Hz). The PLI (Stam et al., 2007) was used to estimate functional connectivity. The PLI is based on the distribution of the differences of the instantaneous phases (derived from the Hilbert transformation of the times series) for two time series, and is computed as:

$$
P L I=\left|\left\langle\operatorname{sign}\left[\sin \left(\Delta \Phi\left(t_{\mathrm{k}}\right)\right)\right]\right\rangle\right|
$$


where " $<>$ " indicates the mean value, "sign" stands the signum function, "I.|" denotes the absolute value and " $t_{\mathrm{k}}$ " are the samples. This measure is not sensitive to volume conduction (at the cost of discarding true zero-lag interactions). PLI values range between 0 and 1 , where 1 indicates perfect synchronization and 0 indicates non synchronous activity. We obtained a $90 \times 90$ adjacency matrix for each epoch for each subject, in all the frequency bands (Stam et al., 2007). For each epoch, the PLI matrix was computed, and after this step they are merged by arithmetic average.

\section{Network Analysis}

The adjacency matrices we obtained were interpreted as networks, where the 90 sources are the nodes and the 1/PLI values are the edges. For each frequency band, a MST was calculated based on each adjacency matrix using Kruskal's algorithm (Kruskal, 1956). The algorithm classifies the links in growing order and, then, builds the network by adding a link at a time. If the link forms a loop, it is discarded. The algorithm proceeds until all nodes are connected resulting in a loop-less binary graph with $N$ nodes and $M=N-1$ links. The MST was used to obtain topological measures that are unaffected by degree distribution, matrix density or arbitrary thresholds (van Wijk et al., 2010).

Based on the MST matrix, we calculated both global and nodal parameters. The former provide insight on the network as a whole. In details, we calculated the leaf fraction (fraction of nodes with degree equal to 1), the tree hierarchy (quantification of the trade-off between node-overload and efficient communication), and the degree divergence (amplitude of the degree distribution; Boersma et al., 2013; Stam et al., 2014; Tewarie et al., 2015). Furthermore, we calculated nodal parameters that provide information on the centrality of each of the 90 ROI. We computed the degree, the betweenness centrality $(\mathrm{BC})$ and the eccentricity. The degree is the number of connections incident on a given node. The $\mathrm{BC}$ represents the number of shortest paths passing through a given node, divided by the total number of shortest paths of the network (Boersma et al., 2013). The eccentricity is defined as the longest path between a node and any other node of the network. The lower the eccentricity, the more central the node (Tewarie et al., 2015). Figure 1 shows the data analysis pipeline (Sorrentino et al., 2018).

\section{Statistical Analysis}

To compare clinical variables between the two groups we applied T-tests. MST metrics were compared, for each frequency band and for each parameter, between the two groups using

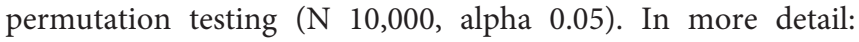
the labels of each metric (i.e., whether a value belongs to the aMCI or controls group) were randomly exchanged 10,000 times. At each iteration we computed the difference between the two groups, building a distribution of the differences. We used this distribution to compute the statistical significance of the experimentally observed groups difference. Then, the false discovery rate (FDR), using the Benjamini-Hochberg procedure (Benjamini and Hochberg, 1995), was applied to correct for multiple comparisons across the global MST measures $(N=3)$, and separately across the 90 ROIs for the nodal measures. The reported $p$-values that follow are always the FDR-corrected values (indicated as $\mathrm{pFDR}$ ).

If a given node was found to have significantly different centrality in the two groups, we compared all the links incident upon that node in order to identify the ones that differed between the two groups, as follows: based on the PLI adjacency matrix, each link was averaged separately for the MCI and control groups, and the average strength was subsequently compared using permutation analysis, as described above. The results were corrected for multiple comparisons across links using FDR. To investigate the topological role of the links incident upon the significantly different nodes, we also checked how many times each link was included in the MST in both MCI and controls. To check if the frequency of inclusion in the MST differed between the two groups, we used a Chi-square test and corrected the results across the links using the FDR.

Finally, to calculate the correlation between clinical variables (neuropsychological scores) and both MRI data (hippocampal volume) and statistically significant network parameters, we used Pearson's correlation coefficients. Correlations were calculated for the aMCI group only, because the neuropsychological scores in the control group showed a too narrow distribution to allow a reliable estimation of the correlation in this group. The linear correlations that have been computed were not corrected for multiple comparisons.

$T$-test and Pearson's correlation coefficients were evaluated using IBM ${ }^{\circledR}$ SPSS Statistics ${ }^{\circledR}$ (version 20). Permutation testing and FDR correction were performed in Matlab (Mathworks ${ }^{\circledR}$, version R2013a). A significance level of $p<0.05$ was used.

\section{RESULTS}

\section{Population Characteristics}

The studied population consists of 16 aMCI patients and $16 \mathrm{HC}$ subjects. Comparing the clinical variables between the two groups, no significant differences were found in age, educational level, gender, depression (Beck Depression Inventory, BDI), language skills (fluency, phrase construction). Significant differences in hippocampal volume, global cognitive status (Mini Mental State Examination, MMSE), memory tests (Free and Cued Selective Reminding Test (FCSRT), Rey words, immediate visual memory), frontal efficiency (Frontal Assessment Battery, FAB) and constructive praxia (freehand copying of drawings, copying drawings with landmarks) were evident. As expected, patients showed worse cognitive performances (Table 2).

\section{MEG Data}

We found several differences between the brain networks in aMCI and HCs. In the theta band, the degree $(p F D R=0.036)$ and the $\mathrm{BC}(p F D R<0.001)$ of the left superior temporal pole (TP) were significantly lower in patients compared to controls. Conversely, in the right middle TP we found higher $\mathrm{BC}$ $(p F D R=0.027)$ in the aMCI group (Figure 2)

For the links incident upon the left superior TP, no individual link differed significantly between the two groups. The right 


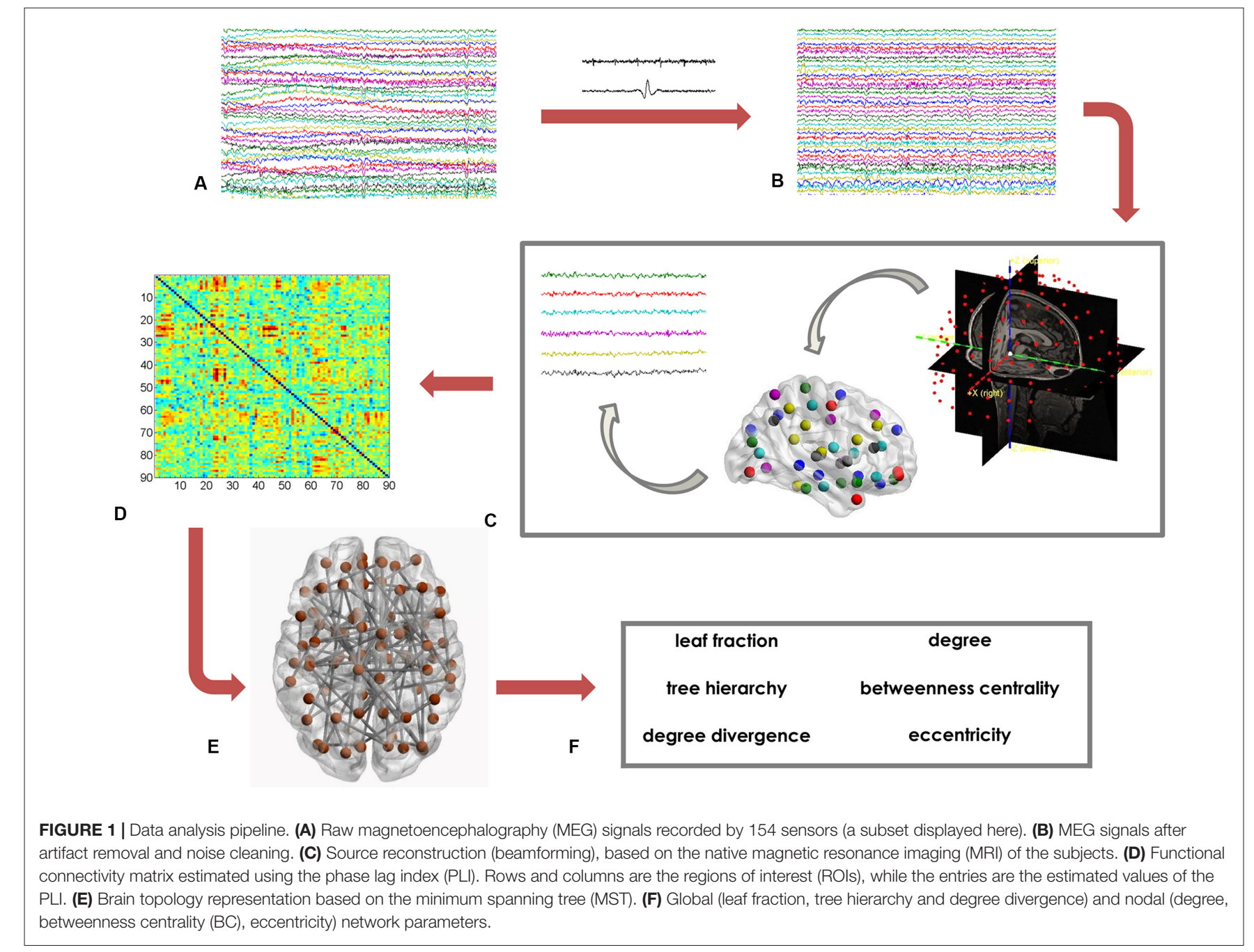

medial TP, however, was more strongly connected to the right cuneus in controls as compared to the MCI population $(p F D R=0.0009)$.
No significant difference was found in the frequency of inclusion in the MST between the two groups for any link incident on the left superior or right middle TP.

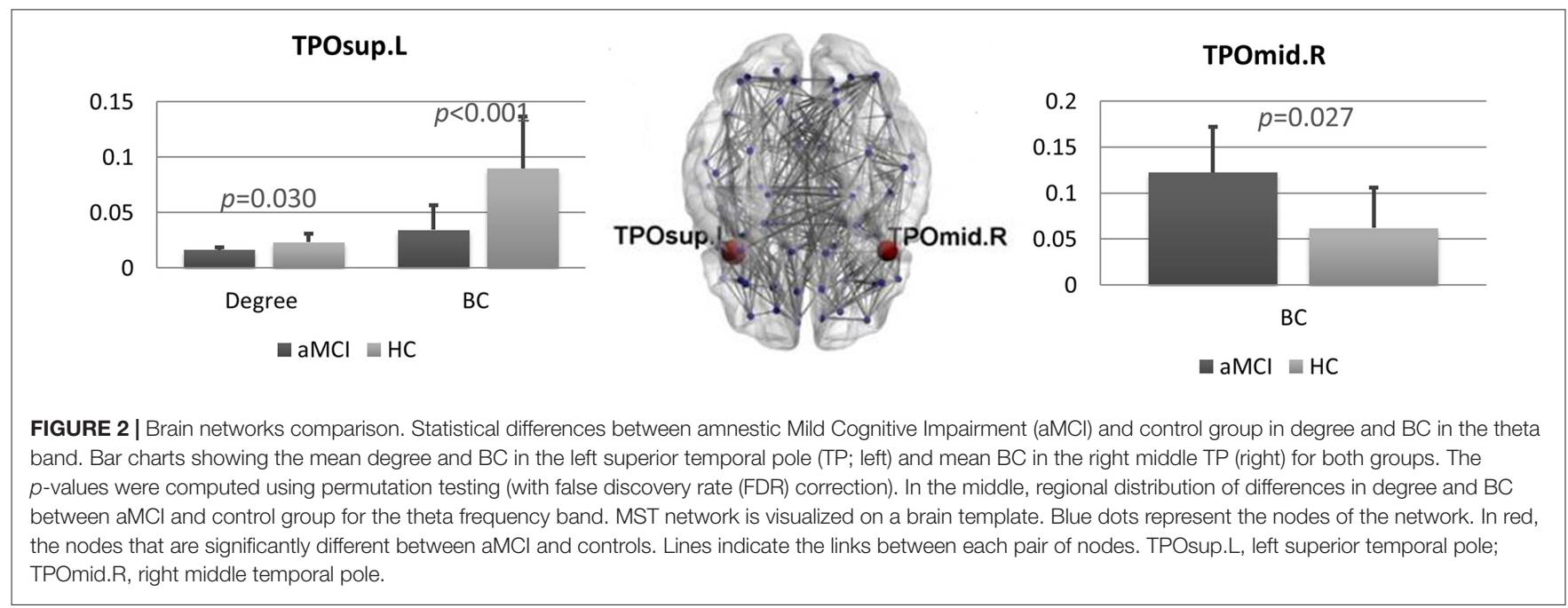


The global MST parameters (leaf fraction, tree hierarchy and degree divergence) did not differ between the two groups. No statistically significant differences were found in any other frequency band (delta, alpha, beta and gamma) for any of the MST parameters.

\section{Correlations}

We calculated the correlations between clinical variables (neuropsychological scores) and both MRI data (hippocampal volume) and statistically significant network parameters. The right hippocampal volume correlated positively with three of the five subtests of the FCSRT (immediate total recall $\left(r_{(21)}=0.546\right.$; $p=0.029)$, delayed total recall $\left(r_{(21)}=0.612 ; p=0.012\right)$ and sensitivity index $\left.\left(r_{(21)}=0.508 ; p=0.044\right)\right)$. In theta band, the $\mathrm{BC}$ of the left superior TP correlated negatively with the FCSRT delayed total recall score $\left(r_{(21)}=0.507 ; p=0.045\right.$; Figure 3).

\section{DISCUSSION}

In the present work, we aimed at identifying subtle changes in network topology in aMCI, which is considered as a prodromal stage of AD. Our hypothesis is that in aMCI the topological alterations of the functional network may be limited to brain regions typically affected by early neurodegeneration and to frequency bands involved in memory processing. To test our hypothesis, we applied the PLI followed by the MST on sourcelevel MEG data obtained from a cohort of aMCI patients compared to HCs.
The two groups were comparable by age, educational level and depression status, while neuropsychological scores, especially memory tests and the hippocampal volume allowed a clear division between aMCI and controls. According to Albert's criteria, given the presence of hippocampal atrophy, the likelihood that in our population the cognitive impairment was due to AD was "intermediate" (Albert et al., 2011). In addition, the observed positive correlation between right hippocampal volume and memory scores is in accordance with the role of hippocampal volume as a progression marker (Frankó et al., 2013). The right hippocampal volume directly correlated with three of the five subtests of the FCSRT (immediate total recall, delayed total recall, Index of Sensitivity of Cueing), confirming the strong link between hippocampal atrophy and memory efficiency (Stoub et al., 2010). It has been shown that the FCSRT test isolates storage disorders due to the involvement of medial temporal structures, that is typical of $\mathrm{AD}$, from other memory deficits related to attention or retrieval disturbances (Frasson et al., 2011). This test has been included in the current diagnostic criteria for $\mathrm{AD}$, since it detects specific episodic memory profiles characterized by low free recall score that is not normalized by cueing (Dubois et al., 2007, 2014). Correlation with the right hemisphere is consistent with the FCSRT visuospatial material used (de Toledo-Morrell et al., 2000) and confirms that our aMCI cohort displays clinical characteristics similar to the ones of the prodromal stage of $\mathrm{AD}$ (Dubois et al., 2014).

The global network parameters that we took into account (MST based leaf fraction, tree hierarchy and degree divergence)

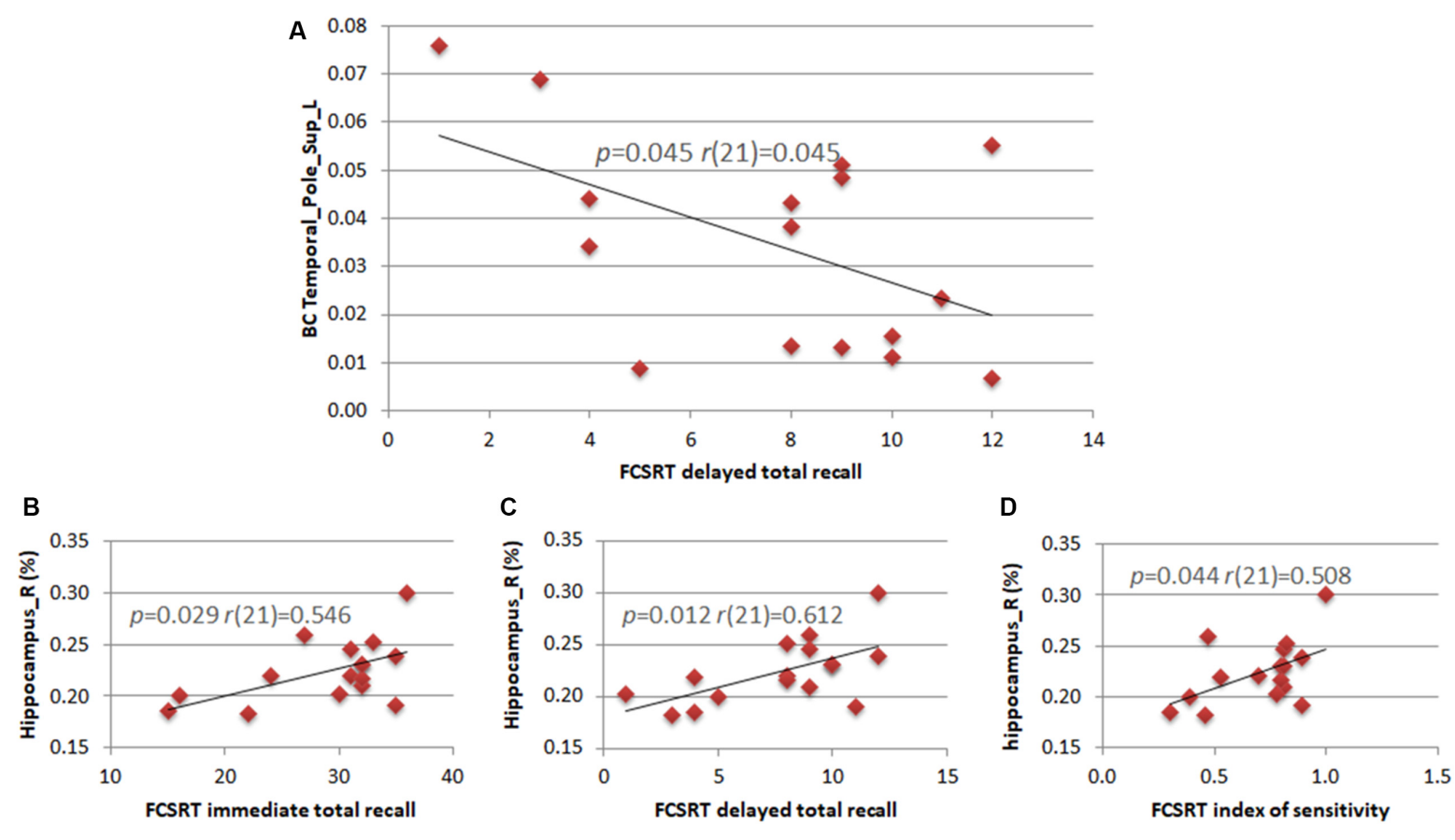

FIGURE 3 | Correlations between neuropsychological scores, hippocampal volumes and network parameters. Pearson's correlation between the BC of the left superior TP and the subtest of the Free and Cued Selective Reminding Test (FCSRT) regarding delayed total recall (A). Pearson's correlation between the right hippocampus volume and subtests of the FCSRT regarding immediate total recall (B), delayed total recall (C) and index of sensitivity (D). 
failed to show any difference between the two groups. AD is characterized by an overall decrease of functional connectivity, a loss of modularity and a specific vulnerability of long-range connections and of the hubs (Tijms et al., 2013). Generally, in MCI the network features might be considered as in an intermediate stage between $\mathrm{AD}$ and healthy subjects (Yao et al., 2010). Increased synchronization between brain areas has been demonstrated in MCI, especially for long-distance connections, as well as reduced modularity, with altered segregation/integration balance (Buldú et al., 2011). In contrast, reduced functional connectivity has recently been reported for medial temporal and parietal regions in MCI compared to HCs (Cuesta et al., 2015). A possible explanation of such incongruence could be found in differences in both the clinical features of the samples and the functional connectivity metric adopted. Only very few MEG studies describe PLI-based networks in $\mathrm{AD}$ and MCI. Interestingly, in these articles alterations of global network parameters have been reported in $\mathrm{AD}$ but not in MCI. Stam et al. (2009) found that in AD, in a no-task, eyes-closed condition, the clustering coefficient and path length in the lower alpha band were both decreased. Furthermore, $\mathrm{Yu}$ et al. (2016), using EEG, found that in the alpha band the global topological features suggested a less integrated topology in AD as compared to controls. Recently, López et al. (2017) focused on brain networks specifically in MCI and found no differences between MCI and controls in terms of global network parameters (averaged normalized weighted clustering coefficient and path length). Our results, obtained with the bias-free MST analysis, are in line with this evidence, indicating that there are no global topological changes in aMCI. Hence, one may speculate that in the very early phase of disease regional changes in functional connectivity (and network topology-see below) may precede global alterations. However, longitudinal studies are needed to confirm this hypothesis.

Comparing the nodal network parameters between groups, we found significant differences in the TPs, in the theta band. The theta rhythm has been linked to memory integration, from the encoding of new information to the retrieval of stored items (for reviews see Kirk and Mackay, 2003; Lisman, 2005). It has been shown that theta power in the medial temporal lobe increases when new memories are included in an existing mnemonic representation (Backus et al., 2016). To this regard, an association between the theta rhythm, $\mathrm{AD}$, and cognitive impairment has been described previously. In fact, higher EEG theta amplitude in parietal, occipital, temporal and limbic areas has been found in $\mathrm{AD}$ (Babiloni et al., 2006) and an increased theta relative power has been described using MEG in MCI patients (López et al., 2014), as well as in early AD in almost all cortical regions, including the hippocampi (Engels et al., 2016). Such evidence shows that the theta band yields information that is specifically relevant for $\mathrm{AD}$ and $\mathrm{MCI}$.

Our data show changes in topological features in the TPs. More specifically, in the left superior TP, degree and BC were significantly lower in aMCI as compared to controls, while in the right middle $\mathrm{TP}$ the $\mathrm{BC}$ was higher in the aMCI group. $\mathrm{BC}$ and degree are both indices of node centrality in the network. The BC reflects the importance of a node for the interactions among the other nodes in the network, and the degree reflects how many connections insist upon a given node. Our results suggest that the functional role of the TPs is modified in aMCI. Such areas are primarily affected in AD (Delacourte et al., 1999; Thompson et al., 2003). Interestingly, the TPs display a central role within memory circuitry, connecting it to further anatomical structures within and beyond the temporal lobe (Olson et al., 2007).

Interestingly, the nodal features of the left and right TPs display an opposite tendency. This result is partially in agreement with López et al. (2017). They found a higher BC in the lower alpha band in the superior and middle gyrus of the right $\mathrm{TP}$, associated with a decreased $\mathrm{BC}$ in upper alpha band in the left middle temporal gyrus. However, none of these findings persisted after FDR correction. The role of the TPs and their functional lateralization has been described as "enigmatic" (Olson et al., 2007). The left TP is mainly considered a semantic hub (Clark et al., 2007), implicated in semantic memory, typically affected in semantic dementia (Landin-Romero et al., 2016). The right $\mathrm{TP}$, given its connections with the amygdala and the orbitofrontal cortex, is involved in emotional control (Chan et al., 2009). By integrating hippocampal information with those from posterior associative areas, the right TP retrieves past emotional experiences to evaluate the meaning of the current stimulus, driving the associated behavioral response (Lane et al., 1999; Nadal, 2013).

Our observation of a "mirrored" change between left and right $\mathrm{TP}$ does not find a univocal interpretation. A recent functional connectivity study between the left and right anterior temporal lobe underlined the interaction between the two poles (Warren et al., 2009). The authors proposed that the inhibition of the injured hemisphere by the intact one could be an adaptive process after stroke. The opposite characteristics that we observed in the two TPs could be related to a similar mechanism, in which one $\mathrm{TP}$ is influenced by the activity of the contralateral one. Alternatively, it is possible that the neurodegenerative process affects the two TPs at different times. It has been proposed that the increased activity and connectivity often recorded in the early phases of the pathological course may be related to a loss of inhibitory connections, subsequently leading to further neuronal damage, loss of connectivity, and network disruption (de Haan et al., 2012). In this line of thinking, the different alterations observed in the TPs in the right and left hemisphere would be due to differences in the staging of the pathological process.

Finally and interestingly, we observed that, in patients, the $\mathrm{BC}$ of the left superior TP showed a negative correlation with the FCSRT delayed total recall. Patients with higher BC show worse memory performances, or vice versa, patients with lower $\mathrm{BC}$ have better memory performances. However, it is not clear what the mechanisms that underlie this phenomenon could be. One might speculate that, when the brain damage is still limited, it might be possible to guarantee long term memory efficiency by lowering the computational load in memory circuitry. When the brain damage is widespread, this mechanism would be no longer efficient and the memory performances would worsen. 
Hence, the inverse relationship between $\mathrm{BC}$ and cognitive scores might be due to overload of the TP related to more widespread brain damage. Recently, de Haan et al. (2012) showed that more central areas tend more easily to metabolic overload and consequent degeneration. Probably, the negative relationship observed between $\mathrm{BC}$ and memory performance could be explained within this framework.

Comparing the PLI values of the links that connect the right middle TP and the left superior TP with other brain regions, the right middle TP appeared to be more weakly connected to the right cuneus in the aMCI group as compared to the control group. This finding is in line with recent evidence showing that posterior links are hypoconnected in MCI as compared to controls, while the opposite happens for anterior links (LópezSanz et al., 2017).

In conclusion, we aimed to identify changes in network properties that could characterize aMCI, which may precede the development of overt AD (Shah et al., 2000; Petersen et al., 2009). Our results indicate that in aMCI there are changes in network centrality with an opposite trend between left and right $\mathrm{TP}$, with no concurrent global network alteration. These changes are frequency-specific in the theta band, which is classically associated with memory processes. Our findings suggest that the pathological process induces changes in the role of the TP within the functional brain networks, and that the extent of these changes is related to the memory performance in aMCI patients.

\section{REFERENCES}

Albert, M. S., DeKosky, S. T., Dickson, D., Dubois, B., Feldman, H. H., Fox, N. C., et al. (2011). The diagnosis of mild cognitive impairment due to Alzheimer's disease: recommendations from the National Institute on Aging-Alzheimer's Association workgroups on diagnostic guidelines for Alzheimer's disease. Alzheimers Dement. 7, 270-279. doi: 10.1016/j.jalz.2011. 03.008

Babiloni, C., Binetti, G., Cassetta, E., Dal Forno, G., Del Percio, C., Ferreri, F., et al. (2006). Sources of cortical rhythms change as a function of cognitive impairment in pathological aging: a multicenter study. Clin. Neurophysiol. 117, 252-268. doi: 10.1016/j.clinph.2005.09.019

Backus, A. R., Schoffelen, J. M., Szebényi, S., Hanslmayr, S., and Doeller, C. F. (2016). Hippocampal-prefrontal theta oscillations support memory integration. Curr. Biol. 26, 450-457. doi: 10.1016/j.cub.2015.12.048

Baillet, S. (2017). Magnetoencephalography for brain electrophysiology and imaging. Nat. Neurosci. 20, 327-339. doi: 10.1038/nn.4504

Benjamini, Y., and Hochberg, Y. (1995). Controlling the false discovery rate: a practical and powerful approach to multiple testing. J. R. Stat. Soc. Series B 57, 289-300.

Boersma, M., Smit, D. J. A., Boomsma, D. I., De Geus, E. J. C., Delemarre-van de Waal, H. A., and Stam, C. J. (2013). Growing trees in child brains: graph theoretical analysis of electroencephalography-derived minimum spanning tree in 5- and 7-year-old children reflects brain maturation. Brain Connect. 3, 50-60. doi: 10.1089/brain.2012.0106

Braak, H., Alafuzoff, I., Arzberger, T., Kretzschmar, H., and Del Tredici, K. (2006). Staging of Alzheimer disease-associated neurofibrillary pathology using paraffin sections and immunocytochemistry. Acta Neuropathol. 112, 389-404. doi: 10.1007/s00401-006-0127-z

Braak, H., and Braak, E. (1991). Neuropathological stageing of Alzheimer-related changes. Acta Neuropathol. 82, 239-259. doi: 10.1007/bf00308809

Braak, H., and Braak, E. (1995). Staging of Alzheimer's disease-related neurofibrillary changes. Neurobiol. Aging 16, 271-278. doi: 10.1016/01974580(95)00021-6

\section{AUTHOR CONTRIBUTIONS}

FJ collected the sample, performed the neuropsychological assessment, performed the MEG recordings, pre-processed the MEG data, wrote the manuscript and prepared the figures. PS collected the sample, performed the MEG recordings, pre-processed the MEG data, performed topological and statistical analysis and wrote the manuscript. AL performed the neuropsychological assessment, performed the MEG recordings and pre-processed the MEG data. RR performed the MEG recordings, pre-processed the MEG data, and contributed to MEG data analysis and statistical analysis. FB collaborated with the MEG data analysis and with the statistical analysis. CC and MA collaborated with the MRI data analysis. MO performed the MRI recordings. AI, VM and AC collected the sample. CG supervised the study. $\mathrm{AH}$ contributed to interpreting the results and critically revised the article. GS supervised the study and wrote the manuscript. All authors read and approved the final version of the manuscript.

\section{FUNDING}

The work was supported by a grant from Regione Campania (Sportello Innovazione; DSMB202) and University of Naples Parthenope "Ricerca locale" (2018).

Braak, H., and Braak, E. (1996). Evolution of the neuropathology of Alzheimer's disease. Acta Neurol. Scand. Suppl. 94, 3-12. doi: 10.1111/j.1600-0404.1996. tb05866.x

Brier, M. R., Thomas, J. B., and Ances, B. M. (2014). Network dysfunction in Alzheimer's disease: refining the disconnection hypothesis. Brain Connect. 4, 299-311. doi: 10.1089/brain.2014.0236

Buckner, R. L., Sepulcre, J., Talukdar, T., Krienen, F. M., Liu, H., Hedden, T., et al. (2009). Cortical hubs revealed by intrinsic functional connectivity: mapping, assessment of stability, and relation to Alzheimer's disease. J. Neurosci. 29, 1860-1873. doi: 10.1523/jneurosci.5062-08.2009

Buldú, J. M., Bajo, R., Maestú, F., Castellanos, N., Leyva, I., Gil, P., et al. (2011). Reorganization of functional networks in mild cognitive impairment. PLoS One 6:e19584. doi: 10.1371/journal.pone.0019584

Carlesimo, G. A., Caltagirone, C., and Gainotti, G. (1996). The Mental Deterioration Battery: normative data, diagnostic reliability and qualitative analyses of cognitive impairment. Eur. Neurol. 36, 378-384. doi: 10.1159/000117297

Chan, D., Anderson, V., Pijnenburg, Y., Whitwell, J., Barnes, J., Scahill, R., et al. (2009). The clinical profile of right temporal lobe atrophy. Brain 132, 1287-1298. doi: 10.1093/brain/awp037

Clark, D. L., Boutros, N. N., and Mendez, M. F. (2007). The Brain and Behavior: An Introduction to Behavioral Neuroanatomy. (Cambridge: Cambridge University Press), 681-683.

Cuesta, P., Garcés, P., Castellanos, N. P., López, M. E., Aurtenetxe, S., Bajo, R., et al. (2015). Influence of the APOE $\varepsilon 4$ allele and mild cognitive impairment diagnosis in the disruption of the MEG resting state functional connectivity in sources space. J. Alzheimers Dis. 44, 493-505. doi: 10.3233/JAD-141872

de Cheveigné, A., and Simon, J. Z. (2007). Denoising based on time-shift PCA. J. Neurosci. Methods 165, 297-305. doi: 10.1016/j.jneumeth.2007.06.003

de Haan, W., Mott, K., van Straaten, E. C., Scheltens, P., and Stam, C. J. (2012). Activity dependent degeneration explains hub vulnerability in Alzheimer's disease. PLoS Comput. Biol. 8:e1002582. doi: 10.1371/journal.pcbi.1002582

de Toledo-Morrell, L., Dickerson, B., Sullivan, M. P., Spanovic, C., Wilson, R., and Bennett, D. A. (2000). Hemispheric differences in hippocampal 
volume predict verbal and spatial memory performance in patients with Alzheimer's disease. Hippocampus 10, 136-142. doi: 10.1002/(SICI)10981063(2000) 10:2<136::AID-HIPO2>3.0.CO;2-J

Delacourte, A., David, J. P., Sergeant, N., Buée, L., Wattez, A., Vermersch, P., et al. (1999). The biochemical pathway of neurofibrillary degeneration in aging and Alzheimer's disease. Neurology 52, 1158-1165. doi: 10.1212/WNL.52. 6.1158

Dubois, B., Feldman, H. H., Jacova, C., DeKosky, S. T., Barberger-Gateau, P., Cummings, J., et al. (2007). Research criteria for the diagnosis of Alzheimer's disease: revising the NINCDS-ADRDA criteria. Lancet Neurology. 6, 734-746. doi: 10.1016/S1474-4422(07)70178-3

Dubois, B., Feldman, H. H., Jacova, C., Hampel, H., Molinuevo, J. L., Blennow, K., et al. (2014). Advancing research diagnostic criteria for Alzheimer's disease: the IWG-2 criteria. Lancet Neurol. 13, 614-629. doi: 10.1016/S14744422(14)70090-0

Engels, M. M. A., Hillebrand, A., van der Flier, W. M., Stam, C. J., Scheltens, P., and van Straaten, E. C. W. (2016). Slowing of hippocampal activity correlates with cognitive decline in early onset Alzheimer's disease. Front. Hum. Neurosci. 10:238. doi: 10.3389/fnhum.2016.00238

Engels, M. M. A., van der Flier, W. M., Stam, C. J., Hillebrand, A., Scheltens, P., and van Straaten, E. C. W. (2017). Alzheimer's disease: the state of the art in resting-state magnetoencephalography. Clin. Neurophysiol. 128, 1426-1437. doi: 10.1016/j.clinph.2017.05.012

Fazekas, F., Chawluk, J. B., Alavi, A., Hurtig, H. I., and Zimmerman, R. A. (1987). MR signal abnormalities at $1.5 \mathrm{~T}$ in Alzheimer's dementia and normal aging. Am. J. Roentgenol. 149, 351-356. doi: 10.2214/ajr.149.2.351

Fischl, B., Salat, D. H., Busa, E., Albert, M., Dieterich, M., Haselgrove, C., et al. (2002). Whole brain segmentation: automated labeling of neuroanatomical structures in the human brain. Neuron 33, 341-355. doi: 10.1016/S08966273(02)00569-X

Frankó, E., Joly, O., and Alzheimer's Disease Neuroimaging Initiative. (2013). Evaluating Alzheimer's disease progression using rate of regional hippocampal atrophy. PLoS One 8:e71354. doi: 10.1371/journal.pone.00 71354

Fraschini, M., Demuru, M., Puligheddu, M., Floridia, S., Polizzi, L., Maleci, A., et al. (2014). The re-organization of functional brain networks in pharmacoresistant epileptic patients who respond to VNS. Neurosci. Lett. 580, 153-157. doi: 10.1016/j.neulet.2014.08.010

Frasson, P., Ghiretti, R., Catricalà, E., Pomati, S., Marcone, A., Parisi, L., et al. (2011). Free and cued selective reminding test: an italian normative study. Neurol. Sci. 32, 1057-1062. doi: 10.1007/s10072-011-0607-3

Friston, K. J. (2011). Functional and effective connectivity: a review. Brain Connect. 1, 13-36. doi: 10.1089/brain.2011.0008

Gong, G., He, Y., Concha, L., Lebel, C., Gross, D. W., Evans, A. C., et al. (2009). Mapping anatomical connectivity patterns of human cerebral cortex using in vivo diffusion tensor imaging tractography. Cereb. Cortex 19, 524-536. doi: 10.1093/cercor/bhn102

Gross, J., Baillet, S., Barnes, G. R., Henson, R. N., Hillebrand, A., Jensen, O., et al. (2013). Good practice for conducting and reporting MEG research. Neuroimage 65, 349-363. doi: 10.1016/j.neuroimage.2012.10.001

Gross, J., Schmitz, F., Schnitzler, I., Kessler, K., Shapiro, K., Hommel, B., et al. (2004). Modulation of long-range neural synchrony reflects temporal limitations of visual attention in humans. Proc. Natl. Acad. Sci. U S A 101, 13050-13055. doi: 10.1073/pnas.0404944101

Iavarone, A., Ronga, B., Pellegrino, L., Loré, E., Vitaliano, S., Galeone, F., et al. (2004). The frontal assessment battery (FAB): normative data from an Italian sample and performances of patients with Alzheimer's disease and frontotemporal dementia. Funct. Neurol. 19, 191-195.

Jack, C. R. Jr., Knopman, D. S., Jagust, W. J., Shaw, L. M., Aisen, P. S., Weiner, M. W., et al. (2010). Hypothetical model of dynamic biomarkers of the Alzheimer's pathological cascade. Lancet Neurol. 9, 119-128. doi: 10.1016/S1474-4422(09)70299-6

Kanfer, J. N., Sorrentino, G., and Sitar, D. S. (1999). Amyloid $\beta$ peptide membrane perturbation is the basis for its biological effects. Neurochem. Res. 24, 1621-1630. doi: 10.1023/A:1021172620295

Kirk, I., and Mackay, J. (2003). The role of theta-range oscillations in synchronising and integrating activity in distributed mnemonic networks. Cortex 39, 993-1008. doi: 10.1016/s0010-9452(08)70874-8
Kruskal, J. B. (1956). On the shortest spanning subtree of a graph and the traveling salesman problem. Proc. Am. Math. Soc. 7:48-50. doi: 10.1090/s0002-99391956-0078686-7

Landin-Romero, R., Tan, R., Hodges, J. R., and Kumfor, F. (2016). An update on semantic dementia: genetics, imaging, and pathology. Alzheimers Res. Ther. 8:52. doi: 10.1186/s13195-016-0219-5

Lane, R. D., Chua, P. M. L., and Dolan, R. J. (1999). Common effects of emotional valence, arousal and attention on neural activation during visual processing of pictures. Neuropsychologia 37, 989-997. doi: 10.1016/s0028-3932(99)00017-2

Lisman, J. (2005). The theta/ $\gamma$ discrete phase code occuring during the hippocampal phase precession may be a more general brain coding scheme. Hippocampus 15, 913-922. doi: 10.1002/hipo.20121

Lopes da Silva, F. (2013). EEG and MEG: relevance to neuroscience. Neuron 80, 1112-1128. doi: 10.1016/j.neuron.2013.10.017

López, M. E., Cuesta, P., Garcés, P., Castellanos, P. N., Aurtenetxe, S., Bajo, R., et al. (2014). MEG spectral analysis in subtypes of mild cognitive impairment. Age 36:9624. doi: 10.1007/s11357-014-9624-5

López, M. E., Engels, M. M. A., van Straaten, E. C. W., Bajo, R., Delgado, M. L., Scheltens, P., et al. (2017). MEG beamformer-based reconstructions of functional networks in mild cognitive impairment. Front. Aging Neurosci. 9:107. doi: 10.3389/fnagi.2017.00107

López-Sanz, D., Bruña, R., Garcés, P., Martín-Buro, M. C., Walter, S., Delgado, M. L., et al. (2017). Functional connectivity disruption in subjective cognitive decline and mild cognitive impairment: a common pattern of alterations. Front. Aging Neurosci. 9:109. doi: 10.3389/fnagi.2017.00109

Measso, G., Cavarzeran, F., Zappalà, G., Lebowitz, B. D., Crook, T. H., Pirozzolo, F. J., et al. (1993). The mini-mental state examination: normative study of an Italian random sample. Dev. Neuropsychol. 9, 77-85. doi: $10.1080 / 87565649109540545$

Minati, L., Varotto, G., D'Incerti, L., Panzica, F., and Chan, D. (2013). From brain topography to brain topology. Neuroreport 24, 536-543. doi: 10.1097/WNR. 0b013e3283621234

Nadal, M. (2013). The experience of art: insights from neuroimaging. Prog Brain Res. 204, 135-158. doi: 10.1016/B978-0-444-63287-6.00007-5

Nolte, G. (2003). The magnetic lead field theorem in the quasi-static approximation and its use for magnetoenchephalography forward calculation in realistic volume conductors. Phys. Med. Biol. 48, 3637-3652. doi: 10.1088/0031-9155/48/22/002

Olson, I. R., Plotzker, A., and Ezzyat, Y. (2007). The Enigmatic temporal pole: a review of findings on social and emotional processing. Brain 130, 1718-1731. doi: 10.1093/brain/awm052

Oostenveld, R., Fries, P., Maris, E., and Schoffelen, J. M. (2011). FieldTrip: open source software for advanced analysis of MEG, EEG, and invasive electrophysiological data. Comput. Intell. Neurosci. 2011:156869. doi: $10.1155 / 2011 / 156869$

Otte, W. M., van Diessen, E., Paul, S., Ramaswamy, R., Subramanyam Rallabandi, V. P., Stam, C. J., et al. (2015). Aging alterations in whole-brain networks during adulthood mapped with the minimum spanning tree indices: the interplay of density, connectivity cost and life-time trajectory. Neuroimage 109, 171-189. doi: 10.1016/j.neuroimage.2015.01.011

Petersen, R. C., Caracciolo, B., Brayne, C., Gauthier, S., Jelic, V., and Fratiglioni, L. (2014). Mild cognitive impairment: a concept in evolution. J. Intern. Med. 275, 214-228. doi: 10.1111/joim. 12190

Petersen, R. C., Roberts, R. O., Knopman, D. S., Boeve, B. F., Geda, Y. E., Ivnik, R. J., et al. (2009). Mild cognitive impairment: ten years later. Arch. Neurol. 66, 1447-1455. doi: 10.1001/archneurol.2009.266

Petersen, R., Smith, G., Waring, S., Ivnik, R. J., Tangalos, E. G., and Kokmen, E. (1999). Mild cognitive impairment: clinical characterization and outcome. Arch. Neurol. 56, 303-308. doi: 10.1001/archneur.56.3.303

Pievani, M., de Haan, W., Wu, T., Seeley, W. W., and Frisoni, G. B. (2011). Functional network disruption in the degenerative dementias. Lancet Neurol. 10, 829-843. doi: 10.1016/s1474-4422(11)70158-2

Rombetto, S., Granata, C., Vettoliere, A., and Russo, M. (2014). Multichannel system based on a high sensitivity superconductive sensor for magnetoencephalography. Sensors 14, 12114-12126. doi: 10.3390/s140712114

Rosenblum, M., Pikovsky, A., and Kurths, J. (1996). Phase synchronization of chaotic oscillators. Phys. Rev. Lett. 76, 1804-1807. doi: 10.1103/PhysRevLett. 76.1804 
Sadasivan, P. K., and Dutt, D. N. (1996). SVD based technique for noise reduction in electroencephalographic signals. Sign. Process. 55, 179-189. doi: 10.1016/s0165-1684(96)00129-6

Sanz-Arigita, E. J., Schoonheim, M. M., Damoiseaux, J. S., Rombouts, S. A., Maris, E., Barkhof, F., et al. (2010). Loss of 'small-world' networks in Alzheimer's disease: graph analysis of fMRI resting-state functional connectivity. PLoS One 5:e13788. doi: 10.1371/journal.pone.0013788

Shah, Y., Tangalos, E. G., and Petersen, R. C. (2000). Mild cognitive impairment. When is it a precursor to Alzheimer's disease? Geriatrics 55, 65-68.

Sica, C., and Ghisi, M. (2007). "The Italian versions of the beck anxiety inventory and the beck depression inventory-II: psychometric properties and discriminant power," in Leading-Edge Psychological Tests and Testing Research, ed. M. A. Lange (New York, NY: NOVA Science Publishers), 27-50.

Sorrentino, G., and Bonavita, V. (2007). Neurodegeneration and Alzheimer's disease: the lesson from tauopathies. Neurol. Sci. 28, 63-71. doi: 10.1007/s10072-007-0789-x

Sorrentino, P., Iuliano, A., Polverino, A., Jacini, F., and Sorrentino, G. (2014). The dark sides of amyloid in Alzheimer's disease pathogenesis. FEBS Lett. 588, 641-652. doi: 10.1016/j.febslet.2013.12.038

Sorrentino, G., Migliaccio, R., and Bonavita, V. (2008). Treatment of vascular dementia: the route of prevention. Eur. Neurol. 60, 217-223. doi: 10.1159/000151696

Sorrentino, P., Nieboer, D., Twisk, J. W. R., Stam, C. J., Douw, L., and Hillebrand, A. (2017). The hierarchy of brain networks is related to insulin growth factor-1 in a large, middle-aged, healthy cohort: an exploratory magnetoencephalography study. Brain Connect. 7, 321-330. doi: 10.1089/brain. 2016.0469

Sorrentino, P., Rucco, R., Jacini, F., Trojsi, F., Lardone, A., Fabio, B., et al. (2018). Brain functional networks become more connected as amyotrophic lateral sclerosis progresses: a source level magnetoencephalographic study. Neuroimage Clin. 20, 564-571. doi: 10.1016/j.nicl.2018.08.001

Sporns, O. (2011). Networks of the Brain. Cambridge, MA: MIT Press.

Sporns, O. (2013). The human connectome: origins and challenges. Neuroimage 80, 53-61. doi: 10.1016/j.neuroimage.2013.03.023

Sporns, O., Tononi, G., and Kötter, R. (2005). The human connectome: a structural description of the human brain. PLoS Comput. Biol. 1:e42. doi: 10.1371/journal. pcbi. 0010042

Stam, C. J. (2010). Use of magnetoencephalography (MEG) to study functional brain networks in neurodegenerative disorders. J. Neurol. Sci. 289, 128-134. doi: 10.1016/j.jns.2009.08.028

Stam, C. J. (2014). Modern network science of neurological disorders. Nat. Rev. Neurosci. 15, 683-695. doi: 10.1038/nrn3801

Stam, C. J., de Haan, W., Daffertshofer, A., Jones, B. F., Manshanden, I., van Cappellen van Walsum, A. M., et al. (2009). Graph theoretical analysis of magnetoencephalographic functional connectivity in Alzheimer's disease. Brain 132, 213-224. doi: 10.1093/brain/awn262

Stam, C. J., Nolte, G., and Daffertshofer, A. (2007). Phase lag index: assessment of functional connectivity from multi channel EEG and MEG with diminished bias from common sources. Hum. Brain Mapp. 28, 1178-1193. doi: 10.1002/hbm.20346

Stam, C. J., Tewarie, P., Van Dellen, E., van Straaten, E. C. W., Hillebrand, A., and Van Mieghem, P. (2014). The trees and the forest: characterization of complex brain networks with minimum spanning trees. Int. J. Psychophysiol. 92, 129-138. doi: 10.1016/j.ijpsycho.2014.04.001

Stoub, T. R., Rogalski, E. J., Leurgans, S., Bennett, D. A., and deToledo-Morrell, L. (2010). Rate of entorhinal and hippocampal atrophy in incipient and mild AD: relation to memory function. Neurobiol. Aging 31, 1089-1098. doi: 10.1016/j. neurobiolaging.2008.08.003

Tewarie, P., van Dellen, E., Hillebrand, A., and Stam, C. J. (2015). The minimum spanning tree: an unbiased method for brain network analysis. Neuroimage 104, 177-188. doi: 10.1016/j.neuroimage.2014.10.015

Thompson, P. M., Hayashi, K. M., de Zubicaray, G., Janke, A. L., Rose, S. E., Semple, J., et al. (2003). Dynamics of gray matter loss in Alzheimer's disease. J. Neurosci. 23, 994-1005. doi: 10.1523/JNEUROSCI.23-03-00994.2003

Tijms, B. M., Wink, A. M., de Haan, W., van der Flier, W. M., Stam, C. J., Scheltens, P., et al. (2013). Alzheimer's disease: connecting findings from graph theoretical studies of brain networks. Neurobiol. Aging 34, 2023-2036. doi: 10.1016/j.neurobiolaging.2013.02.020

Trojsi, F., Sorrentino, P., Sorrentino, G., and Tedeschi, G. (2017). Neurodegeneration of brain networks in the amyotrophic lateral sclerosisfrontotemporal lobar degeneration (ALS-FTLD) continuum: evidence from MRI and MEG studies. CNS Spectr. doi: 10.1017/s109285291700075x [Epub ahead of print].

Tzourio-Mazoyer, N., Landeau, B., Papathanassiou, D., Crivello, F., Etard, O., Delcroix, N., et al. (2002). Automated anatomical labeling of activations in SPM using a macroscopic anatomical parcellation of the MNI MRI single-subject brain. Neuroimage 15, 273-289. doi: 10.1006/nimg.2001.0978

Van Veen, B. D., van Drongelen, W., Yuchtman, M., and Suzuki, A. (1997). Localization of brain electrical activity via linearly constrained minimum variance spatial filtering. IEEE Trans. Biomed. Eng. 44, 867-880. doi: 10.1109/10.623056

van Wijk, B., Stam, C., and Daffertshofer, A. (2010). Comparing brain networks of different size and connectivity density using graph theory. PLoS One 5:e13701. doi: 10.1371/journal.pone.0013701

Wang, J. (2010). Graph-based network analysis of resting-state functional MRI. Front. Syst. Neurosci. 4:16. doi: 10.3389/fnsys.2010.00016

Warren, J. E., Crinion, J. T., Lambon Ralph, M. A., and Wise, R. J. S. (2009). Anterior temporal lobe connectivity correlates with functional outcome after aphasic stroke. Brain 132, 3428-3442. doi: 10.1093/brain/awp270

Watts, D., and Strogatz, S. (1998). Collective dynamics of 'small-world' networks. Nature 393, 440-442. doi: 10.1038/30918

Yao, Z., Zhang, Y., Lin, L., Zhou, Y., Xu, C., and Jiang, T. (2010). Abnormal cortical networks in mild cognitive impairment and Alzheimer's disease. PLoS Comput. Biol. 6:e1001006. doi: 10.1371/journal.pcbi.1001006

Yu, M., Gouw, A. A., Hillebrand, A., Tijms, B. M., Stam, C. J., van Straaten, E. C. W., et al. (2016). Different functional connectivity and network topology in behavioral variant of frontotemporal dementia and Alzheimer's disease: an EEG study. Neurobiol. Aging 42, 150-162. doi: 10.1016/j. neurobiolaging.2016.03.018

Conflict of Interest Statement: The authors declare that the research was conducted in the absence of any commercial or financial relationships that could be construed as a potential conflict of interest.

Copyright (C) 2018 Jacini, Sorrentino, Lardone, Rucco, Baselice, Cavaliere, Aiello, Orsini, Iavarone, Manzo, Carotenuto, Granata, Hillebrand and Sorrentino. This is an open-access article distributed under the terms of the Creative Commons Attribution License (CC BY). The use, distribution or reproduction in other forums is permitted, provided the original author(s) and the copyright owner(s) are credited and that the original publication in this journal is cited, in accordance with accepted academic practice. No use, distribution or reproduction is permitted which does not comply with these terms. 